\title{
Correction: Development of G: a test in an amphibious fish
}

\author{
Joseph M. Styga $\left(^{1,2} \cdot\right.$ Thomas M. Houslay $\mathbb{1}^{3,4} \cdot$ Alastair J. Wilson $^{4} \cdot$ Ryan L. Earley ${ }^{2}$
}

Published online: 27 February 2019

(c) The Genetics Society 2019

Correction to: Heredity (2018);

https://doi.org/10.1038/s41437-018-0152-4;

Published online 16 October 2018

Figure 3 legend has been corrected to state: "Difference matrices for pairwise-trait phenotypic correlations (rP, below diagonal) and pairwise-trait genetic correlations ( $\mathrm{rG}$, above diagonal) from 1, 15, and $100 \mathrm{DPH}$. Differences are color coded by strength and direction. Differences shown in gray are positive and differences shown in black are negative. When ages are similar, the colored square is small; when ages are very different, the colored square fills the cell. EPL Epural length, EPA epural angle, PHPL parahypural length,
PHPA parahypural angle, HYPL hypural length, HYPW hypural width, and SL standard length."

Originally, the text for Fig. 3 was printed as such: "Difference matrices for pairwise-trait phenotypic correlations (rP, below diagonal) and pairwise-trait genetic correlations ( $\mathrm{rG}$, above diagonal) from 1, 15, and $100 \mathrm{DPH}$. When ages are similar, the colored square is small; when ages are very different, the colored square fills the cell. EPL epural length, EPA epural angle, PHPL parahypural length, PHPA parahypural angle, HYPL hypural length, HYPW hypural width, and SL standard length."

This has now been corrected in both the PDF and HTML versions of the article.
The original article can be found online at https://doi.org/10.1038/ s41437-018-0152-4.

Joseph M. Styga

jstyga@culver.edu

1 Department of Biology, Culver-Stockton College, One College Hill, Canton, MO 63435, USA

2 Department of Biological Sciences, University of Alabama, 300 Hackberry Lane, Box 870344, Tuscaloosa, AL 35487, USA

3 Department of Zoology, University of Cambridge, Downing Street, Cambridge CB2 3EJ, UK

4 Centre for Ecology and Conservation, University of Exeter-Penryn Campus, Cornwall TR10 9FE, UK 


\section{1 vs $15 \mathrm{DPH}$}

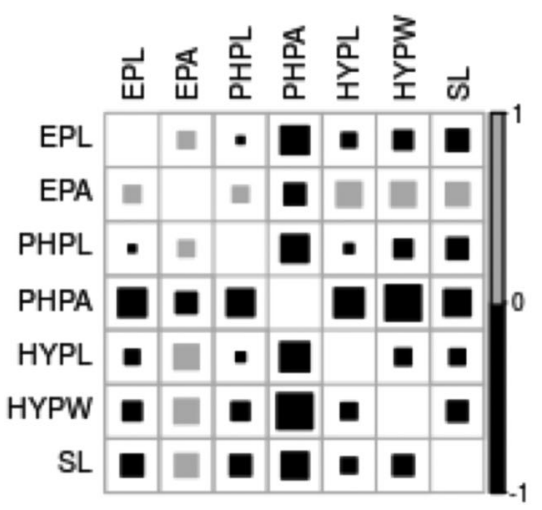

1 vs $100 \mathrm{DPH}$

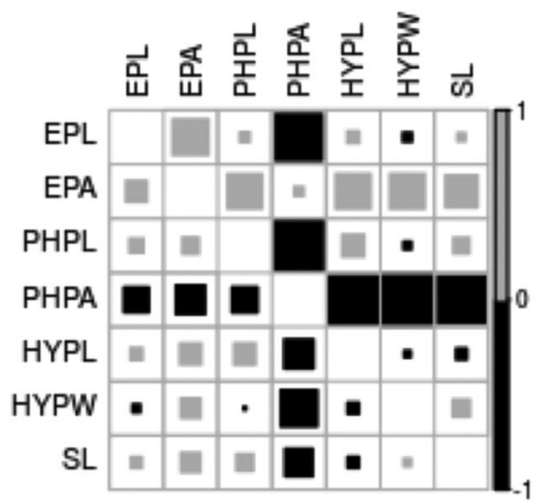

15 vs. $100 \mathrm{DPH}$

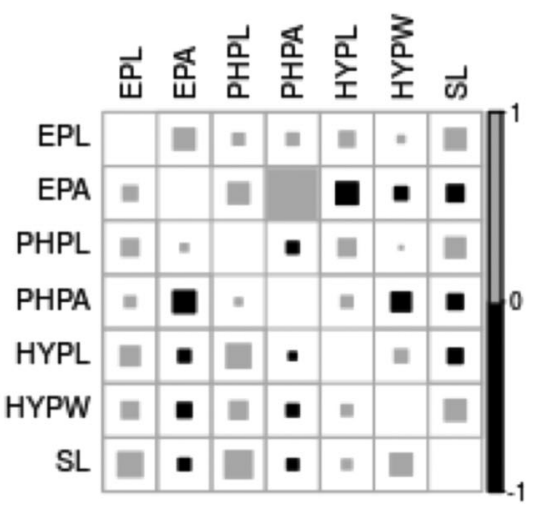

Fig. 3 Difference matrices for pairwise-trait phenotypic correlations ( $\mathrm{rP}$, below diagonal) and pairwise-trait genetic correlations ( $\mathrm{rG}$, above diagonal) from 1, 15, and 100 DPH. Differences are color coded by strength and direction. Differences shown in gray are positive and differences shown in black are negative. When ages are similar, the colored square is small; when ages are very different, the colored square fills the cell. EPL Epural length, EPA Epural angle, PHPL parahypural length, PHPA parahypural angle, HYPL hypural length, HYPW hypural width, and SL standard length 